\title{
Emotional Symptoms and Their Related Factors in Adolescents During the Acute Phase of COVID-19 Outbreak in Southa Italy
}

simone pisano ( $\nabla$ pisano.simone@gmail.com )

Universita degli Studi di Salerno - Campus Baronissi https://orcid.org/0000-0001-5704-4483

Gennaro Catone

Suor Orsola Benincasa University of Naples: Universita degli Studi Suor Orsola Benincasa

Antonella Gritti

Suor Orsola Benincasa University of Naples: Universita degli Studi Suor Orsola Benincasa

Luisa Almerico

University of Campania Luigi Vanvitelli: Universita degli Studi della Campania Luigi Vanvitelli

Anna Pezzella

University of Campania Luigi Vanvitelli: Universita degli Studi della Campania Luigi Vanvitelli

Pia Santangelo

National Specialty Hospital Santobono Pausilipon: Azienda Ospedaliera di Rilievo Nazionale Santobono

Pausilipon

Carmela Bravaccio

Federico II University Hospital: Azienda Ospedaliera Universitaria Federico II

Raffaella Iuliano

Ospedale del Mare

Vincenzo Paolo Senese

University of Campania Luigi Vanvitelli: Universita degli Studi della Campania Luigi Vanvitelli

\section{Research}

Keywords: pandemic, anxiety symptoms, depressive symptoms, youth, quarantine

Posted Date: December 1st, 2020

DOI: https://doi.org/10.21203/rs.3.rs-114475/v1

License: (c) (i) This work is licensed under a Creative Commons Attribution 4.0 International License.

Read Full License 
Version of Record: A version of this preprint was published at Italian Journal of Pediatrics on April 8th, 2021. See the published version at https://doi.org/10.1186/s13052-021-01036-1. 


\section{Abstract}

\section{Background}

Several studies have shown that during COVID-19 pandemic outbreak, emotional symptoms increased in the general population. Less is known in youths.

\section{Methods}

We surveyed a sample of Italian adolescents during the strictest quarantine period and assessed the effects of socio-demographic and psychological factors on current emotional symptoms. A convenient sample of 326 adolescents (age range 14-19 years) participated in a web-based survey. We collected data on several socio demographic and psychological information (summarized into three indexes: environmental context, changes in lifestyle, and worries about infection) and psychopathological symptoms (previous psychopathological status, current anxiety and depressive symptoms).

\section{Results}

Descriptive analysis showed that adolescents have experienced quarantine under very different conditions; they reported $47.5 \%$ and $14.1 \%$ of anxiety and depressive symptoms, respectively. Regression analyses indicated that previous psychopathological status and worries about infection are linked to anxiety and that female gender, previous psychopathological status (moderated by change in lifestyle), worse environmental context are linked to depression.

\section{Conclusion}

This study indicates that, facing the COVID-19 pandemic and its related safety measures, adolescents show relevant emotional symptoms and therefore should be monitored, assessed and supported.

\section{Background}

Since March 2020, Italy has been facing the COVID-19 outbreak and this soon after China, slightly before other world countries. Italian government had enacted a strict quarantine in the attempt to reduce the contagion. Fear of own and relatives' contagion and worries for physical health join social distancing and isolation, as well as economic difficulties representing a wealth of highly distressing factors [1]. For example, it is well known that quarantine itself may produce negative psychological effects including post-traumatic stress symptoms, confusion, and anger [2]. It is likely that suicide and other dangerous behaviours may increase [1,3]. A recent review revealed a high prevalence of psychiatric symptoms in general population during COVID-19 pandemic and pointed out that we all are still unaware of long term outcomes [4]. There is a clear consensus among experts that mental health status during pandemic outbreaks should be assessed and mental health problems addressed $[1,5]$. This is particularly true regarding fragile populations such as health workers, psychiatric patients and youths $[1,2,6]$. We here focus the latter. Adolescents may face stressors such as fears of infection, frustration and boredom, 
inadequate information, lack of in-person contact with classmates, friends, and teachers, lack of personal space at home, and family financial loss worse than adults [7]. The routes to psychiatric disorders usually pass through adverse events and traumas. Thus, it is imperative to empirically study the psychopathological effects of a pandemic outbreak, such as the COVID-19, and of its related safety measures, such as social distancing and quarantine. This, in fact, would allow planning possible interventions to prevent negative psychological consequences in the event of future pandemics. In a recent study focusing the adolescent Chinese population, Zhou et al. [8] revealed a prevalence of depressive symptoms, anxiety symptoms, and a combination of depressive and anxiety symptoms during COVID-19 outbreak of $43.7 \%, 37.4 \%$, and $31.3 \%$, respectively, much higher than estimates usually reported in China in pre-COVID era. Another study, not limited to adolescents, but including young adults, reported that nearly $40 \%$ of youths are prone to psychological problems [9]. In Italy, a study on adults reported a high prevalence of those suffering from high or very high levels of distress, with females being more susceptible [10]. Another study on adults reported that about $38 \%$ displayed mild to severe likelihood of psychological distress, with cyclothymic, anxious and depressive temperaments being risk factors [11]. Here we aimed at expanding these findings, providing more evidence in adolescents, and contextually thoroughly assessing environmental and psychological factors that may be linked to the raise of emotional symptoms (state anxiety and depressive symptoms). We collected the data between April 25th and May 13th 2020, which strictly correspond to the highest level of social restrictions imposed by Italian Government (full lockdown; see also [12]), in order to catch the putative psychological impact of both strictest quarantine and exposure to pandemic outbreak. We hypothesize a higher prevalence of emotional symptoms during this period compared to previously reported ones. Also, based on previous studies $[13,14]$, we hypothesize that a previous general tendency to psychopathology, unfavourable environmental and acute psychological changes such as an uncomfortable and "not connected" quarantine living context, acute lifestyle changes (in sleep and eating habits) and higher worries about COVID-19 could be factors related to emotional symptoms.

\section{Methods}

\subsection{Participants}

The recruitment of participants was school-based. Schools were chosen based on previous contact between them and the research team, their previous experience on epidemiological surveying, on their known compliance to mental health research and their availability in organize an expedite dissemination of the survey. Among four schools contacted, three agreed to participate; they are placed in the metropolitan area of Naples, in Campania region, south of Italy; they all are professional institutes that, culturally, in Italy, are more frequented by males and by low/mid income families.

Before the data collection, participants' parents and each adolescents had the opportunity to carefully read an informative sheet about the research that the schools had provided and to ask for questions to teachers and researchers; then, parents and youths were requested to agree in participating in the study through an online informed consent form. A total of 472 subjects responded to the school invitation. Of 
these, $412(87.3 \%)$ gave their consent to the study, and 326 (79.1\%) completed the whole survey and were included in the analyses. The final sample included 247 males $(75.8 \%$, age range $14-19$ years, $M$ age $=$ $15.8, S D=1.3)$ and 79 females (24.2\%, age range $14-19$ years, $M$ age $=16.0, S D=1.4)$. Males and females did not differ on age, $t(324)=1.20, p=231$. We lack data on those who did not gave their consent. The study was approved by the Ethical Committee of the "A.O.R.N. Antonio Cardarelli - A.O.R.N. Santobono-Pausilipon" Hospital of Naples (Italy).

\subsection{Procedures}

The study was conducted through the Qualtrics online survey platform between April 25th and May 13th 2020. In this period, schools disseminated to their students a link to the online platform that presented the survey. The data described here are those collected cross-sectionally. The whole study is expected to be longitudinal with a new collection of data within six months (follow-up) that will include longitudinal outcomes. To this end, each participant has been assigned an anonymous alpha-numeric code that will allow us to link the answers to the follow-up data collection. We did not apply any exclusion criteria.

As regards the cross-sectional part presented here, the survey included two sections: (a) a sociodemographic questionnaire (SQ) devised ad hoc and investigating the living contest and quarantine conditions of participants, everyday lifestyle habits and worries about COVID-19 (see supplementary file); and (b) a section including mental health standardized measures: State-Trait Anxiety Inventory (STAl), Mood and Feelings Questionnaire-short form (MFQ-SF) and Strength and Difficulties Questionnaire (SDQ).

\subsection{Measures}

Socio-demographic Questionnaire (SQ). A socio-demographic questionnaire asking gender, age, the characteristics of the dwelling (i.e., number of rooms, presence of private room, open spaces, internet. etc.), the changes in everyday lifestyle habits related to the quarantine (e.g., sleep, feeding, etc.), and worries about COVID-19 (e.g. afraid of possible own and relative's infection), was administered. The answers collected in this section have been used to create three indexes able to measure the live context of the participants during the quarantine and investigate the relationship of these indexes with the psychological effects of the pandemic: (1) environmental context (EC), is an index extracted from the responses related to the participants' living space and its potential comfort; higher scores on this measure indicate that participants had more space available in their homes, open spaces accessible, a good internet connection and a good device to connect to the internet; (2) changes in lifestyle (CL), is an index extracted from the responses related to the participants' changes in everyday lifestyle habits; higher scores on this measure indicate higher changes in the quality and quantity of feeding habits and sleep; (3) worries about infection (WI), is an index extracted from participants' responses related to worries about themselves and their close family member of being exposed to the COVID-19 infection; higher scores on this measure indicate that participants had higher worries and tendency to collect information about the contagion. 
State-Trait Anxiety Inventory (STAI). The Italian version of State-Trait Anxiety Inventory (STAI, form Y) [15, $16]$ is a commonly used measure of anxiety, with good psychometric properties. The STAI form $Y$ has 40 items, 20 items developed to measure the state anxiety (S-A) and 20 items developed to measure the trait anxiety (T-A). Items of the S-A scale assess intensity of current feelings ("at this moment") and are rated on a 4-point scale: 1 = "not at all", 2 = "somewhat", 3 = "moderately", and 4 = "very much". Items of the T-A scale assess frequency of feelings "in general" and are rated on a 4-point scale: 1 "almost never", 2 = "sometimes", 3 = "often", and 4 = "almost always". For both scales, higher scores indicate greater anxiety, state or trait respectively. In the present paper, only the S-A was administered as a measure of current anxiety symptoms. Observed Cronbach's alphas was .912. A value of 40 can be considered as a clinical threshold value (cut-off) predictive of anxiety symptoms, distinguishing three levels of severity: mild (from 40 to 50), moderate (from 51 to 60) and severe (scores greater than 61) [17].

Mood and Feelings Questionnaire-short form (MFQ-SF). The self-report child short form of the Mood and Feelings Questionnaire [18, 19] was used to assess current depressive symptoms. The MFQ-SF includes 13 items that cover depressive symptoms over the past 2 weeks. It is a widely used instrument for the screening of depressive symptoms in the general population of adolescents, with good psychometric properties. In the present study, the original 13-item MFQ-SF was translated into Italian by bilingual psychologists and then back-translated by two independent translators to verify the equivalence of the translated scale to the original one [20]. An exploratory factorial analysis (Principal Factor Analysis) carried out on the scale confirmed the unidimensional structure; eigenvalues (and percentage of explained variance) of the first three factors were respectively $5.76(44.3 \%), 1.19(9.2 \%)$ and $0.96(7.4 \%)$. For the purpose of the present study, a total score was computed. Higher scores indicate greater depression. In this study, Cronbach's alpha was .887. Angold and colleagues pointed out that, in the short version of the scale, the value of 12 can be considered as a clinical threshold value (cut-off) predictive of depressive symptomatology [18].

Strength and Difficulties Questionnaire (SDQ). The Italian self-report version of the Strength and Difficulties Questionnaire (SDQ)21 was administered to assess the general psychopathology. The SDQ includes 25 items, related to five domains: (1) emotional symptoms ( 5 items), (2) conduct problems (5 items), (3) hyperactivity-inattention problems ( 5 items), (4) peer problems (5 items), and (5) prosocial behaviour (5 items). Individual items are scored on a 3-point scale, 0 = "not true"; 1 = "somewhat true"; and 2 = "certainly true", with a score range 0-10 for each subscale. The psychometric properties of the self-report version of the SDQ are generally acceptable to good across studies [22]. For the purpose of the present study, we used the total score (excluding the prosocial behaviour scale) as a measure of general psychopathology referring to the last six months. Higher scores indicate greater psychopathological symptoms. In this study, Cronbach's alpha was .738. A four band categorization of the total score was suggested: "close to the average" (from 0 to 14), "slightly raised" (from 15 to 17), "high" (from 18 to 19) and "very high" (from 20 to 40) [23].

\subsection{Statistical analyses}


Descriptive statistics were firstly used to describe demographics and data related to the living contest during the quarantine, the lifestyle changes and worries about COVID-19, the state anxiety (STAI S-A), the depressive symptoms (MFQ-SF) and the general psychopathology symptoms (SDQ). The frequency distribution of responses and/or means and standard deviation of variables were reported. Then, responses to items within each of the three considered domains, environmental context (EC), changes in lifestyle (CL) and worries about infection (WI), were analysed to extract a single indicator useful to summarize the several questions of the sections. Once extracted, the association between the three indicators and emotional symptoms controlling for previous general psychopathological status was investigated. To this aim, two hierarchical multiple regression analyses were carried out on the state anxiety scores (STAI S-A) and the depression scores (MFQ-SF) respectively. In both models, in the first step, the gender (dummy coded: male $=1$, female $=0$ ) and age ( $z$-score) were included as control variables, in the second step, the SDQ score (z-score) was included as the independent variables, in the third step the three indicators (z-scores) were included as the independent variables, whereas in the final fourth step, the two-way interaction effects were considered to test the moderation effect of the three stress indicators on the relation between the SDQ and the considered dependent variables.

\section{Results}

\subsection{Socio-demographic section}

Environmental Context (EC). Analyses of responses collected in this section of the protocol showed that, with regard to the place of residence (item 1), $73.6 \%$ of the participants experienced quarantine in a dwelling with four or more rooms (excluding bathrooms and closets, $M=4.3, S D=1.3$ ). Most of the adolescents did not have a room for themselves (item 2), but shared their own room with a brother/sister (53.4\%), while $32.5 \%$ had their own room to study and sleep in. With regard to open spaces (item 3), $3.7 \%$ had no outdoor space available in the house (only windows) or in the condominium, $5 \%$ had only a condominium space available (courtyard or green area), $46.3 \%$ had a balcony, while $45.1 \%$ had a terrace or garden. Regarding the possibility to access the Internet (item 4), the data showed that $3.1 \%$ did not have access to the Internet, 9.5\% accessed the Internet with their mobile phone or for a limited time, 3.1\% accessed the Internet via Wi-Fi but for a limited time, 58.6\% accessed the Internet via unlimited time Wi-Fi, while $25.8 \%$ accessed the Internet via fast and unlimited time Wi-Fi. With regard to the availability of devices to connect to the Internet (item 5), the data showed that $33.7 \%$ had a mobile phone only, $3.1 \%$ had a tablet, $36.2 \%$ a shared PC and $27.0 \%$ a PC for exclusive use. To get a single measure of environmental context (variable named EC), each of the five items of the section were recorded into three levels of comfort (low, medium and high) and a principal component analysis (PCA) was carried out by extracting one single component and computing the factor score. The PCA showed that the unidimensional solution explained $30.7 \%$ of the variance, with each item showing a saturation $>.497$. The higher was the score on this index, the better was the living context during the lockdown.

Changes in lifestyle (CL). Analyses of responses collected in this section of the protocol showed that there were changes in both eating habits and sleep-wake rhythms during the quarantine. In particular, 
with regard to eating habits, the data showed that $82 \%$ of adolescents stated that they had modified their diet from a quantitative point of view (item 1, 54.0\% "a little", $28.2 \%$ "a lot"). 57.9\% stated that they had modified their diet from a qualitative point of view (item 2, 42.9\% "a little", 15.0\% "a lot"). $68.4 \%$ declared to have modified the times (hours and frequency) of the alimentation (item 3, 43.2\% "a little", 25.2\% "a lot"). With regard to the analysis of changes in biological rhythms and sleep quality, more generalized changes emerge. In fact, $65.0 \%$ of adolescents declared that they have modified "a lot" the sleep-wake rhythms (item 4), 29.4\% "a little", while only 5.5\% "not at all". While, with regard to the quality of sleep (item 5), $40.5 \%$ of adolescents reported that they have modified the quality of sleep "very much", $37.7 \%$ "a little", while only $21.8 \%$ "not at all". To get a single measure of changes in lifestyle (variable named CL), a principal component analysis (PCA) was carried out on the five items of the sections by extracting one single component and computing the factor score. The PCA showed that the unidimensional solution explained $44.2 \%$ of the variance, with each item showing a saturation $>.594$. The higher was the score on this index, the higher were the changes in the lifestyle during the lockdown.

Worries about infection (WI). Analyses of responses collected in this section of the protocol showed that during the quarantine, adolescents were more worried about their families getting infected (item 1), $M=$ 7.2, $S D=3.1$, than they were worried about themselves (item 2), $M=4.3, S D=3.6$; the comparison between these two scores is significant, $t(325)=14.71, p<.001$. In particular, $38.0 \%$ declared they were not worried about contracting the virus themselves, while only $10 \%$ reported they were not worried about a family member contracting the virus. In the latter case, $31 \%$ declared a maximum level of concern, i.e. "10". Finally, with regard to the time spent reading or listening to information related to contagion (item 3 ), the majority of adolescents (50\%) reported they spend about one hour, $13.2 \%$ spend two or more hours, while $12.9 \%$ do not know anything at all. To get a single measure of worries about infection (variable named $\mathrm{WI})$, a principal component analysis (PCA) was carried out on the three items of the sections by extracting one single component and computing the factor score. The PCA showed that the unidimensional solution explained $53.1 \%$ of the variance, with each item showing a saturation $>.589$. The higher was the score on this index, the higher were the worries about the infection during the lockdown.

\subsection{Psychopathology section}

\section{State anxiety}

the assessment of state anxiety symptoms during the COVID-19 revealed that adolescent had a mean score of $41.6(S D=10.8)$; considering the cut-off of 40 , predictive of clinically relevant symptoms 17 , data showed that the $47.5 \%$ of the sample exceeded it; specifically, $27.0 \%$ showed "mild anxiety", $14.1 \%$ showed "moderate anxiety" and 6.4 "severe anxiety". A significant gender difference was observed, $t(324)$ $=5.74, p<.001$, with females showing higher state-anxiety (S-A) than males (see Table 1 ).

\section{Depression}

the assessment of depressive symptoms during the COVID-19 revealed that adolescent had a mean score of $6.5(S D=5.6)$; considering the cut-off of 12 , predictive of clinically relevant symptoms 18 , data showed 
that $14.1 \%$ of the sample exceeded it. A significant gender difference was observed, $t(324)=6.89, p$ $<.001$, with females showing higher depression (MFQ-SF) than males (see Table 1).

\section{General psychopathology}

the assessment of the presence of general psychopathology symptoms referred to the six months (thus before the onset of pandemic) showed that adolescents had a mean total score of $11.4(S D=5.9)$; considering a cut-off score of 14 , data indicate that $26.7 \%$ of the sample exceeded it; specifically, $9.2 \%$ showed a "slightly raised" score, $6.1 \%$ showed a "high" score, $11.3 \%$ showed a "very high" score. A significant gender difference was observed, $t(324)=5.80, p<.001$, with females showing more symptoms (SDQ) than males (see Table 1).

\subsection{Relationship between extracted indicators (EC, CL and WI) and emotional symptoms}

Data from the hierarchical regression analysis are reported in Table 2. Results showed a similar pattern of effects for the two considered dependent variables. As regards the state anxiety, data showed that over and above the control variables (gender and age), the general psychopathology symptoms (SDQ) were uniquely associated with the anxiety scores, $R 2 \mathrm{diff}=.294, p<.001$. As expected, the model-fit increased when the three indicators were entered into the model, $R 2 \mathrm{diff}=.019, p=.017$, whereas the last step did not show significant two-way interaction effects. The parameters of the final model revealed that general psychopathology symptoms (SDQ), $\beta=.556, p<.001$ and worries about infection (WI), $\beta=.110, p=.013$ were both uniquely independent predictors of anxiety, $R 2=.425, p<.001$. No other significant effects were observed. That is, over and above the other variables in the model, the more were the general psychopathology symptoms before the COVID-19 the higher was the state anxiety during the lock-down, the higher was the worries about the infection the more was the anxiety.

As regards the depression, data showed that over and above the control variables (gender and age), the general psychopathology symptoms (SDQ) were uniquely associated with the depression scores, $R 2$ diff $=.390, p<.001$. As expected, the model-fit increased when the three stress indicators were entered into the model, $R 2$ diff $=.020, p=.003$, whereas the last step showed significant two-way interaction effects between indicators and the general psychopathology, $R 2 \mathrm{diff}=.023, p<.001$. The parameters of the final model revealed that gender, $\beta=-.103, p=.012$, general psychopathology symptoms (SDQ), $\beta=.625, p$ $<.001$, environmental context (EC), $\beta=-.106, p=.005$, and changes in lifestyle (CL), $\beta=.108, p=.006$ were all uniquely independent predictors of depression, $R 2=.569, p<.001$, and that the amount of changes in lifestyle $(\mathrm{CL})$ moderated the relation between the general psychopathology and the depression scores (see Fig. 1). No other two-way interaction effects were observed. That is, over and above the other variables in the model, females showed a higher level of depression than males, the more were the general psychopathology symptoms before the COVID-19 the higher was the depression during the lockdown, the better was the living context the lower were the depressive symptoms, the higher were the changes in lifestyle the more was the depressive symptoms as well as the combination of the general psychopathology and changes in lifestyle increased the depressive symptoms. 


\section{Discussion}

We here assessed emotional symptoms and their related factors in adolescents during the strict quarantine period due to COVID-19 pandemic in Italy. We found a prevalence of anxiety and depressive symptoms of respectively $47.5 \%$ and $14.1 \%$ of the sample. These estimates frankly exceeded the previously reported ones in Italy, assessed in a context without any pandemic, via two previous epidemiological studies; in fact, Frigerio et al. [24] found a prevalence of internalizing disorders of $6.5 \%$ and Gritti et al. [25] found a prevalence of internalizing symptoms (in clinical range) of $10.5 \%$. Compared to other studies performed during quarantine period in China, estimates are closer, although with some notable differences. Regarding anxiety, Zhou et al. [8] found very similar percentages (43.7\%), whereas regarding depression their values were higher (37.4\%). Cultural aspects or differences in measurement instruments and/or sample sizes may account for this discrepancy. Compared to studies on the general population, using as reference estimates reported in Xiong et al. [4] who summarize the most reliable ones (using those reported by better appraised studies, e.g. those with the most representative samples), as well as a recent large survey on Italian general adult population [26], ours are higher for anxiety and similar for depression (in their review anxiety symptoms range from $6.3-18.7 \%$ and depressive symptoms range from $14.6-32.8 \%$ [4]; similarly, Fiorillo reported $12.4 \%$ of depressive symptoms and $17.6 \%$ of anxiety symptoms [26]). Globally, our findings add evidence of the enhancing need of psychological and psychiatric care for adolescents during the sanitary emergency; more specifically, although in need of replication with further data, the notion that adolescents are a fragile population, more prone to anxiety symptoms than adults, is confirmed. Efforts to mitigate psychological burden should be a priority for policymakers and health organizers.

We extend our investigations to factors related to emotional symptoms. We wanted to be as inclusive as possible extracting several empirical factors that maybe be theoretically linked to anxiety and depression [13] and assessing previous psychopathological status with a standardized measure (SDQ). The results of our regression analysis showed that more general psychopathological symptoms and higher worries for infection predict heightened anxiety; whereas that female gender, more general psychopathological symptoms, worse environmental quarantine context and changes in lifestyle habits (moderated by psychopathological symptoms) predict heightened depression. Thus, we confirm that subjects suffering from previous psychopathological symptoms are more prone to suffer from an increase of emotional symptoms during the pandemic [26]; more in depth, worries for infection (own and relative's infection) are linked to anxiety symptoms, whereas subjects, especially females, living in unfavourable environmental context and who experienced changes in lifestyle habits are more prone to suffer from depression. Highlighting these characteristics seems important to direct more intensive and more tailored prevention and treatment strategies to the right targets. Social, socio-economic and health support should be firstly directed to people who meet these characteristics.

The study presents some limitations that should be highlighted. First of all, the sample is relatively small, skewed towards males and we lack a rigorous epidemiological sampling methodology. This because we wanted to gather data during the acute phase of pandemic outbreak and when the quarantine was 
strictest, thus the short window of available time prevented a wider dissemination of the online survey. The study then lacked a proper epidemiological sampling of the schools. Those that accepted to participate in the study were all professional institutes, that, as already said, in our region are mostly representative of low-medium socio-economic conditions, with a higher male prevalence. This is reflected by the low number of females in our sample and the lack of objective data on socio economic status; accordingly, firm conclusions on gender differences cannot be drawn and the generalizability to the general population is limited. On the other hand, this let us to gather in a short period an adequately sized sample to test our hypothesis; this is the only study that was able to gather data during a full lockdown period. Also, as all the data were self-reported and rely on an online survey, our study may suffer from social desirability bias or other sampling biases. Ideally, multi informant surveys and/or cross check with personal assessment should be performed to gather data less prone to such bias, but this was impossible during a quarantine period. Of course, our study is cross sectional in nature and thus conclusion on causality should be confirmed with longitudinal ones. Based on previous studies, we focused our study on internalizing symptoms, but it is likely that externalizing ones increase as well; future studies should assess both and their interplay.

Notwithstanding these relevant limitations, although in need of replication and expansion, we can provide useful data to be translated for a better services organization and more targeted policy decisions. Adolescents need to be supported and protected with any efforts during the sanitary emergency due to COVID-19; emotional symptoms are substantially higher compared to a "normal" period and have to be monitored, assessed and detected; those with previous psychopathological symptoms are even more fragile and in need of social and health support.

\section{Abbreviations}

SQ

socio demographic questionnaire; STAI:State-Trait Anxiety Inventory; MFQ-SF:Mood and Feelings Questionnaire-short form; SDQ:Strength and Difficulties Questionnaire; EC:environmental context; $\mathrm{CL}$ :changes in lifestyle; WI:worries about infection.

\section{Declarations}

The study was approved by the Ethical Committee of the "A.O.R.N. Antonio Cardarelli - A.O.R.N. Santobono-Pausilipon" Hospital of Naples (Italy).

Parents and youths consented their participation to the study through an online consent form.

Conflict of interest: the authors declare that they have no competing interests

The datasets used and/or analysed during the current study are available from the corresponding author on reasonable request. 


\section{Author Contributions}

SP, GC: conception and design of the study; draft part of the manuscript.

AP, LA, VPS: acquisition and analysis of data, critically revised the manuscript

$A G, C B, P S, R I$ : draft part of the manuscript, critically revised the manuscript

\section{References}

1. Holmes EA, O'Connor RC, Perry VH et al. Multidisciplinary research priorities for the COVID-19 pandemic: a call for action for mental health science. Lancet Psychiatry. 2020; 7: 547-560. https://doi.org/10.1016/S2215-0366(20)30168-1.

2. Brooks SK, Webster RK, Smith LE et al. The psychological impact of quarantine and how to reduce it: rapid review of the evidence. Lancet. 2020; 395: 912-920. https://doi.org/10.1016/S01406736(20)30460-8.

3. Yip PS, Cheung YT, Chau PH, Law YW. 2010. The impact of epidemic outbreak: the case of severe acute respiratory syndrome (SARS) and suicide among older adults in Hong Kong. Crisis. 2010; 31: 86-92. doi: 10.1027/0227-5910/a000015.

4. Xiong J, Lipsitz O, Nasri F et al. Impact of COVID-19 pandemic on mental health in the general population: A systematic review. J Affect Disord. 2020; 277: 55-64. https://doi.org/10.1016/j.jad.2020.08.001.

5. Gritti A, Salvati T, Russo K, Catone G. COVID-19 pandemic: a note for psychiatrists and psychologists. JPS Journal [Internet]. 29 maggio 2020 [citato 16 novembre 2020];4(1):63 - 77. Available at: https://jpsjournal.org/new/ojs/index.php/JPS/article/view/70.

6. Iasevoli F, Fornaro M, D'Urso $\mathrm{G}$ et al. Psychological distress in serious mental illness patients during the COVID-19 outbreak and one-month mass quarantine in Italy. Psychol Med. 2020; :1-3. https://doi.org/10.1017/S0033291720001841.

7. Wang G, Zhang Y, Zhao J, Zhang J, Jiang F. Mitigate the effects of home confinement on children during the COVID-19 outbreak. Lancet. 2020; 395(10228): 945-947. doi:10.1016/S01406736(20)30547-X.

8. Zhou SJ, Zhang LG, Wang LL et al. Prevalence and socio-demographic correlates of psychological health problems in Chinese adolescents during the outbreak of COVID-19. Eur Child Adolesc Psychiatry. 2020; 29: 749-758. https://doi.org/10.1007/s00787-020-01541-4.

9. Liang L, Ren H, Cao R et al. The Effect of COVID-19 on Youth Mental Health. Psychiatr Q. 2020; 91: 841-852. https://doi.org/10.1007/s11126-020-09744-3.

10. Mazza C, Ricci E, Biondi S et al. A Nationwide Survey of Psychological Distress among Italian People during the COVID-19 Pandemic: Immediate Psychological Responses and Associated Factors. Int J Environ Res Public Health. 2020; 17: e3165. https://doi.org/10.3390/ijerph17093165. 
11. Moccia $L$, Janiri $D$, Pepe $M$ et al. Affective temperament, attachment style, and the psychological impact of the COVID-19 outbreak: an early report on the Italian general population. Brain Behav Immun. 2020; 87: 75-79. https://doi.org/10.1016/j.bbi.2020.04.048.

12. lafusco $M$, Ciampa $C$, De Maddi $F$ et al. PediaVirus chatline: all together against COVID-19 [published online ahead of print, 2020 Jul 20]. Arch Dis Child. 2020; archdischild-2020-319551. doi:10.1136/archdischild-2020-319551.

13. Singh S, Roy D, Sinha K, Parveen S, Sharma G, Joshi G. Impact of COVID-19 and lockdown on mental health of children and adolescents: A narrative review with recommendations. Psychiatry Res. 2020; 293:113429. doi:10.1016/j.psychres.2020.113429.

14. Nikolaidis A, Paksarian D, Alexander $L$ et al. The Coronavirus Health and Impact Survey (CRISIS) reveals reproducible correlates of pandemic-related mood states across the Atlantic. MedRxiv. 2020. doi: $10.1101 / 2020.08 .24 .20181123$.

15. Pedrabissi L, Santinello M. STAI State-Trait Anxiety Inventory Forma Y Manuale. Organizzazioni Speciali, Firenze. 1989.

16. Spielberger CD, Gorsuch RL, Lushene R, Vagg PR, Jacobs GA. Manual for the State-Trait Anxiety Inventory. Consulting Psychologists Press, Palo Alto CA. 1983.

17. Barisone MG, Lerda S, Ansaldi S, De Vincenzo E, Angelini G. Psychopathology and epilepsy: clinical experience in a Centre for the Diagnosis and Care of epilepsy. Ital J Psychopathol. 2004; 10, 3.

18. Angold A, Costello EJ, Messer SC, Pickles A, Winder F, Silver D. The development of a short questionnaire for use in epidemiological studies of depression in children and adolescents. Int $J$ Methods Psychiatr Res. 1995; 5: 237-249.

19. Messer SC, Angold A, Costello EJ, Loeber R, Van Kammen W, Stouthamer-Loeber M. Development of a short questionnaire for use in epidemiological studies of depression in children and adolescents: Factor composition and structure across development. Int J Methods Psychiatr Res. 1995; 5 (4): 251262. Available at: https://nsuworks.nova.edu/cps_facarticles/628.

20. Van de Vijver FJR, Tanzer NK. Bias and equivalence in cross-cultural assessment: An overview. Eur Rev Appl Psychol. 2004; 54(2): 119-135.

21. Goodman R. The Strengths and Difficulties Questionnaire: a research note. J Child Psychol Psychiatry. 1997; 38: 581-586. https://doi.org/10.1111/j.1469-7610.1997.tb01545.x.

22. Van Roy B, Veenstra M, Clench-Aas J. Construct validity of the five-factor Strengths and Difficulties Questionnaire (SDQ) in pre-, early, and late adolescence. J Child Psychol Psychiatry. 2008 Dec; 49(12): 1304-12. doi: 10.1111/j.1469-7610.2008.01942.x.

23. Youth in Mind, 2016. Information for researchers and professionals about the Strengths \& Difficulties Questionnaires. https://www.sdqinfo.org/ (accessed 10 September 2020).

24. Frigerio A, Rucci P, Goodman R et al. Prevalence and correlates of mental disorders among adolescents in Italy: the PrISMA study. Eur Child Adolesc Psychiatry. 2009; 18: 217-26. doi: 10.1007/s00787-008-0720-x. 
25. Gritti A, Bravaccio C, Signoriello S. Epidemiological study on behavioural and emotional problems in developmental age: prevalence in a sample of Italian children, based on parent and teacher reports. Ital J Pediatr. 2014 Feb 18; 40(1):19. doi: 10.1186/1824-7288-40-19.

26. Fiorillo A, Sampogna G, Giallonardo V et al. Effects of the lockdown on the mental health of the general population during the COVID-19 pandemic in Italy: Results from the COMET collaborative network. Eur Psychiatry. 2020; 63(1), e87. https://doi.org/10.1192/j.eurpsy.2020.89.

\section{Tables}

Table 1 Descriptive statistics of the main variables as a function of the gender

\begin{tabular}{|c|c|c|c|c|c|c|c|c|c|}
\hline \multicolumn{4}{|c|}{ Females } & \multicolumn{3}{|c|}{ Males } & \multicolumn{3}{|c|}{$\begin{array}{l}\text { Total } \\
\text { sample }\end{array}$} \\
\hline Variable & $M$ & $S D$ & $\begin{array}{l}\% \\
\text { above cut- } \\
\text { off }\end{array}$ & $M$ & $S D$ & $\begin{array}{l}\% \\
\text { above cut- } \\
\text { off }\end{array}$ & $M$ & $S D$ & $\begin{array}{l}\% \\
\text { above } \\
\text { cut-off }\end{array}$ \\
\hline Age & 16.0 & 1.4 & प & 15.8 & 1.3 & प & 15.9 & 1.3 & $\square$ \\
\hline SDQ & 14.6 & 6.2 & 46.8 & 10.4 & 5.4 & 20.2 & 11.4 & 5.9 & 26.7 \\
\hline $\begin{array}{l}\text { STAI S- } \\
A\end{array}$ & 47.4 & 11.9 & 70.9 & 39.8 & 9.7 & 40.1 & 41.6 & 10.8 & 47.5 \\
\hline MFQ-SF & 10.1 & 6.3 & 29.1 & 5.4 & 4.9 & 9.3 & 6.5 & 5.6 & 14.1 \\
\hline
\end{tabular}

Table 2 Results of the regression analysis (final models) exploring the impact of hypothesized explanatory variables on emotional symptoms 


\begin{tabular}{|lllllll|}
\hline \multicolumn{3}{|l}{ State Anxiety } & \multicolumn{5}{l|}{ Depression } \\
\hline Variable & $b$ & beta & $p$-value & $b$ & beta & $p$-value \\
\hline Males & -0.198 & -0.085 & 0.067 & -0.239 & -0.103 & 0.012 \\
\hline Age & 0.077 & 0.077 & 0.074 & 0.016 & 0.016 & 0.672 \\
\hline SDQ & 0.556 & 0.556 & 0.000 & 0.625 & 0.625 & 0.000 \\
\hline EC & 0.010 & 0.010 & 0.810 & -0.106 & -0.106 & 0.005 \\
\hline CL & 0.082 & 0.082 & 0.065 & 0.108 & 0.108 & 0.006 \\
\hline WI & 0.110 & 0.110 & 0.013 & 0.044 & 0.044 & 0.254 \\
\hline EC $\times$ SDQ & $\square$ & $\square$ & $\square$ & -0.067 & -0.066 & 0.086 \\
\hline CL $\times$ SDQ & $\square$ & $\square$ & $\square$ & 0.138 & 0.139 & 0.000 \\
\hline WI $\times$ SDQ & $\square$ & $\square$ & $\square$ & 0.003 & 0.003 & 0.928 \\
\hline & & & & & & \\
\hline
\end{tabular}

Note $\mathrm{N}=326 ;{ }^{\wedge}$ Males $=$ Gender of participants (dummy coded: male $=1$, female $=0$ ); Age = Age of participants (years; z-score); SDQ = Strength and Difficulties Questionnaire total score (z-score); EC = environmental context index (z-score); $\mathrm{CL}=$ changes in lifestyle index (z-score); $\mathrm{WI}=$ worries about infection index (z-score).

\section{Figures}




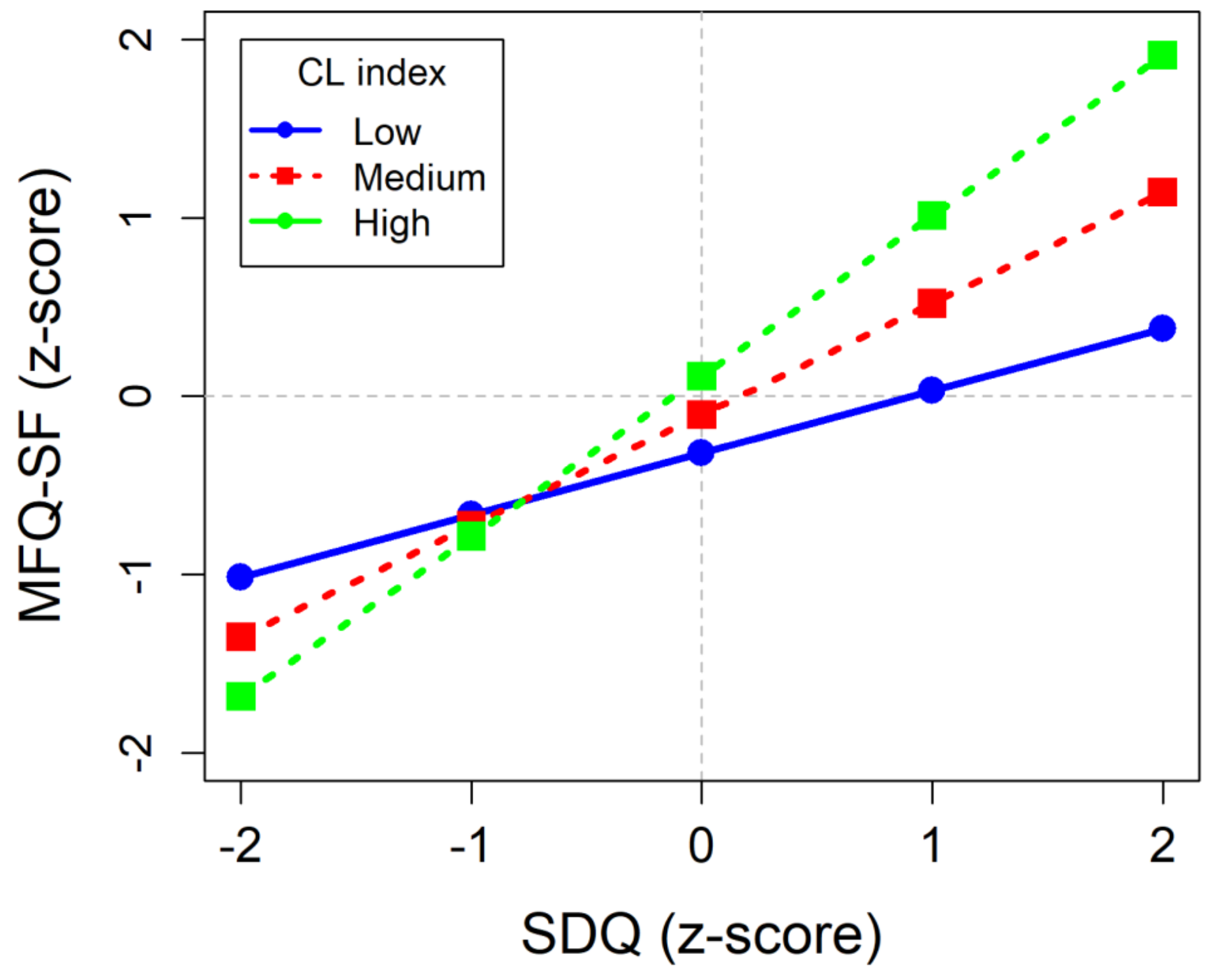

Figure 1

Example of moderation effect of the $\mathrm{CL}$ index on the association between SDQ and the MFQ-SF. To depict the figure, the other consider variables of the model (Male, Age, EC and WI) were fixed to 


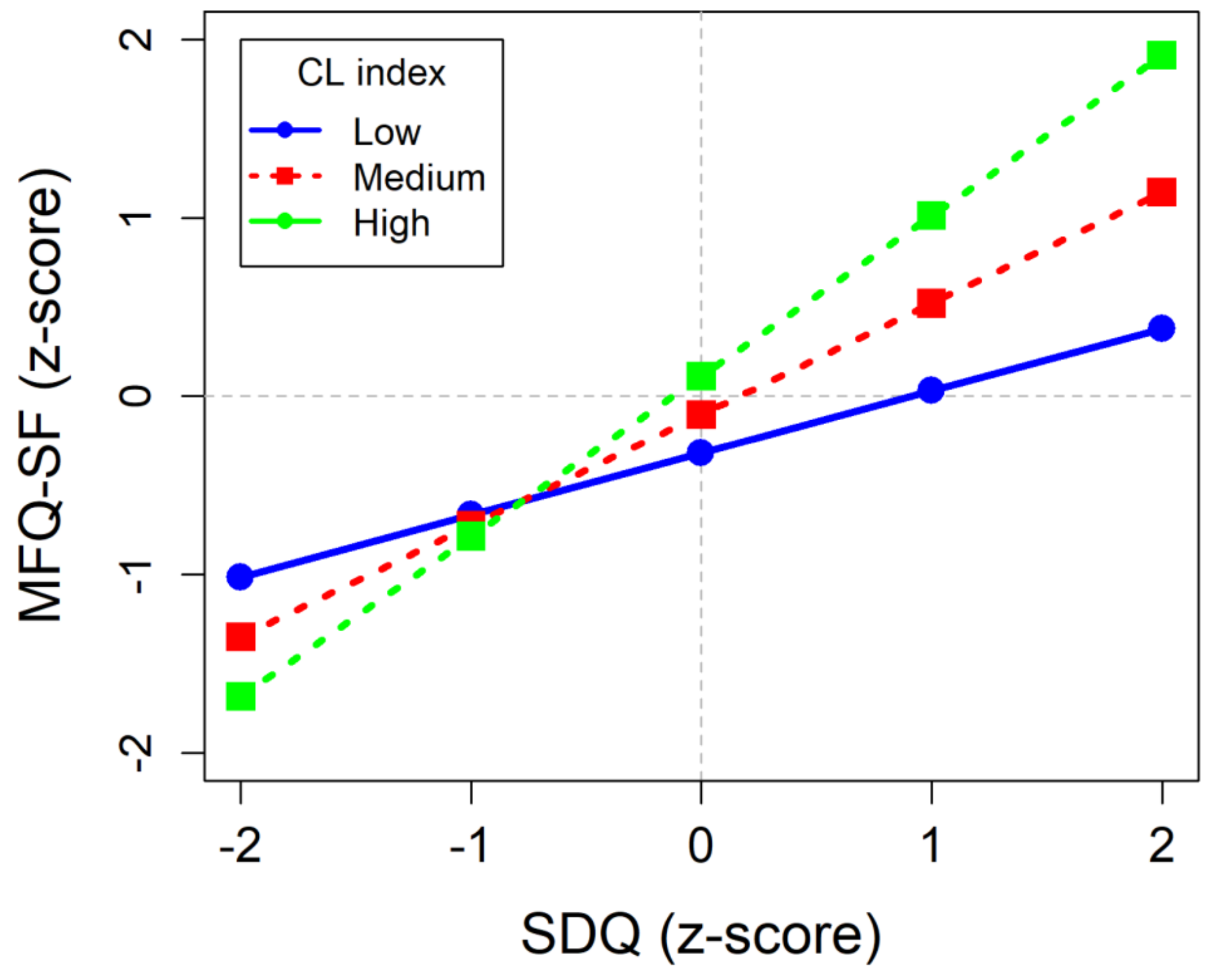

Figure 1

Example of moderation effect of the $\mathrm{CL}$ index on the association between SDQ and the MFQ-SF. To depict the figure, the other consider variables of the model (Male, Age, EC and WI) were fixed to

\section{Supplementary Files}

This is a list of supplementary files associated with this preprint. Click to download.

- Pisanoetal.COVIDadditionalfile1.doc

- Pisanoetal.COVIDadditionalfile1.doc 